\title{
Corela
}

Cognition, représentation, langage

HS-10 | 2012

Paramétrer le sens ? Études de cas

\section{Introduction : Le paramétrage linguistique du sens : pour une linguistique des interfaces}

\section{Martine Sekali}

\section{CpenEdition}

\section{Journals}

Édition électronique

URL : http://journals.openedition.org/corela/2514

DOI : $10.4000 /$ corela.2514

ISSN : $1638-573 \mathrm{X}$

\section{Éditeur}

Cercle linguistique du Centre et de I'Ouest - CerLICO

\section{Référence électronique}

Martine Sekali, «Introduction : Le paramétrage linguistique du sens : pour une linguistique des interfaces », Corela [En ligne], HS-10 | 2012, mis en ligne le 30 janvier 2012, consulté le 01 mai 2019. URL : http://journals.openedition.org/corela/2514; DOI : 10.4000/corela.2514

Ce document a été généré automatiquement le 1 mai 2019.

\section{(c) (i) (2)(2)}

Corela - cognition, représentation, langage est mis à disposition selon les termes de la licence Creative Commons Attribution - Pas d'Utilisation Commerciale - Partage dans les Mêmes Conditions 4.0 International. 


\title{
Introduction : Le paramétrage linguistique du sens : pour une linguistique des interfaces
}

\author{
Martine Sekali
}

\section{Objectifs}

1 La polysémie lexicale comme grammaticale des signes linguistiques est un phénomène avéré. Le lexème français 'thème', selon les contextes de son emploi, pourra ainsi prendre le sens de 'sujet de débat', 'traduction en langue étrangère', 'information ancienne' etc., de même que le marqueur anglais 'since', à la fois polysémique et transcatégoriel, peut instruire une interprétation temporelle (depuis) ou argumentative (puisque). Bien entendu une approche consistant à simplement lister toutes les valeurs référentielles que peuvent construire les signes de la langue ne dit rien du processus leur construction.

2 Les théories de l'énonciation (et en particulier la Théorie des Opérations Prédicatives et Enonciatives définie par A. Culioli) ont ainsi fourni des outils précieux permettant la recherche et la formalisation des opérations invariantes dont les marqueurs linguistiques sont la trace, à travers la multiplicité des représentations construites. De très nombreuses études, depuis les années 1960 ont permis d'accéder à ces opérations au sein de divers systèmes de langue. A partir de ces opérations invariantes, la construction du sens ne peut être retracée que dans les contextes de sa production effective. Cette dépendance du sens au 'contexte', à moins de demeurer une sorte de processus mystérieux ou trop complexe pour être analysé, se doit néanmoins elle aussi d'être formalisée, ou du moins interrogée, investiguée, définie.

3 Qu'entend-on exactement par 'contexte', qu'il soit dit étroit ou large? Quels éléments spécifiques (ou 'variables') dans le contexte d'un énoncé ou d'un texte/discours interagissent avec les opérations invariantes dont les marqueurs linguistiques sont la trace pour construire des valeurs référentielles? Une fois défini un ensemble d'opérations et de variables, comment s'organise cette coopération de signes et 
d'opérations linguistiques dans l'élaboration du sens? Quels niveaux (syntaxique, lexical, grammatical, phonologique, discursif etc.), ou dimensions de la langue, prendre en compte dans l'analyse linguistique ? Ces 'niveaux', qui sont des niveaux métalinguistiques utiles à l'analyse, ont-ils chacun une réalité, ou une pertinence fonctionnelle autonome? Autant de problématiques qu'une démarche de paramétrage du sens se doit de poser, et de soumettre à l'analyse, pour décrire et expliquer le processus de déploiement et de construction du sens, et son/ses interprétation(s) dans des énoncés authentiques.

Il faut bien admettre aussi que la recherche des opérations invariantes des unités linguistiques n'annule en rien la polysémie de ces unités, pas plus d'ailleurs qu'elle n'explique le processus même de l'élaboration des valeurs référentielles. Comme le soulignent très justement Franckel et Paillard (1998) :

«Mais cette approche engendre un paradoxe : si l'opération propre à chaque unité est invariante, et n'aboutit à des résultats variables qu'en fonction des termes pris comme opérandes, la variation se trouve indéfiniment reportée sur le co-texte. Or il se trouve que tout élément du co-texte est lui-même déformable et polysémique, susceptible à son tour de prendre plusieurs valeurs. C'est pourquoi, dans les développements actuels de la théorie, est prise en compte l'idée centrale que la variation est constitutive de l'identité même d'une unité. La notion de marqueur d'opération se trouve ainsi dépassée, dans la mesure où le rapport entre opérateur et opérande est lui-même variable. Il s'avère que l'opérateur n'est pas fixe, il est travaillé de façon spécifique par des facteurs de son environnement. C'est à une interaction dynamique que l'on a affaire, et non à un conditionnement à sens unique. » (Franckel et Palliard 1998, p. 61).

L'intégration de la variation à la schématisation des opérations invariantes a ainsi ouvert la voie à un nouveau modèle de représentation, plus récent, et très porteur dans une démarche de paramétrage du sens, à travers la recherche des " formes schématiques " des unités linguistiques (voir en particulier De Vogüe et Paillard 1997).

6 A nouveau, le regard du linguiste se porte donc aussi sur la nature et sur les caractéristiques de ces 'variables' contextuelles, avec des problématiques essentielles, relevant de leur inscription en creux dans les formes schématiques des unités linguistique: les variables contextuelles sont-elle des données, ou elles-mêmes des fonctions de certaines variables? Doit-on, peut-on, faire le départ entre les opérations et les variables, alors même que les 'variables' co-textuelles sont elles-mêmes opérations, et vice versa, et que c'est dans l'interaction dynamique des opérations, qui s'inter-modifient, que se construit le sens ? Et encore : l'éventail des variables avec lesquelles les opérateurs peuvent interagir est-il totalement ouvert, ou au contraire constitue-t-il un paradigme fermé, et donc mesurable, paramétrable ?

7 D'où l'introduction du terme 'paramètre' : le paramètre, c'est un locus d'interaction opérationnelle, un type (ou famille) de variables convocables par l'unité linguistique, la description formelle de sa 'capacité intégrative' (cf. de Vogüé, ce numéro, pour une discussion des retombées épistémologiques de ce concept).

\section{Démarche}

8 Le paramétrage du sens est une démarche d'analyse linguistique, et non une théorie. Plus précisément, c'est une démarche nécessairement trans-théorique.

9 Cette démarche est double. Paramétrer le sens, c'est d'abord tenter d'élucider le processus dynamique complexe de son élaboration dans les corpora où il se réalise. Le 
sens est ici considéré en tant qu'ensemble de valeurs référentielles, sorte de précipité linguistique issu d'une synergie opérationnelle de structures linguistiques. Le paramétrage du sens vise alors à définir cette synergie linguistique, et à décrire les effets des interactions d'opérations et de paramètres dans le processus d'élaboration du sens tel qu'il se déploie au sein d'un énoncé, d'un discours, d'un texte.

Cette synergie linguistique n'est cependant pas un phénomène totalement ouvert, et les unités linguistiques (ou marqueurs), ne sauraient interagir entre elles indifféremment, de sorte que chaque unité, au sein d'un système de langue, contient dans son schéma opérationnel même un principe de déformabilité (ou de variance) propre (cf. Culioli 1990), et se conjugue potentiellement avec un paradigme fermé (bien que large) d'autres opérations linguistiques. C'est ce paradigme que nous désignons par le terme de 'paramètres'. C'est la définition de ces principes (ou schémas) de déformabilité que nous envisageons dans une démarche de paramétrage du sens des unités linguistiques.

11 Cette démarche vise donc aussi, par retour, à anticiper les potentialités de la réalisation du sens en contexte, en intégrant la polysémie des unités linguistiques à leur invariance opérationnelle au sein d'un système de langue. Il s'agit donc en particulier de formaliser (et de circonscrire) des familles de paramètres qui, bien que n'étant pas tous réalisés au sein des énoncés, sont inscrits en creux dans le schéma de fonctionnement des marqueurs de la langue. Paramétrer le sens (entité complexe et mouvante), qu'il soit potentiel ou réalisé, stabilisé ou non, c'est donc aussi tenter de cerner les principes de son élaboration dans les systèmes de langue.

\section{Une linguistique des interfaces}

12 Le sens étant considéré moins comme compositionnel que multidimensionnel et dynamique, le paramétrage relève d'une démarche d'analyse qui impose que l'on travaille sur les interfaces linguistiques.

\section{Interface entre les formes schématiques}

13 Les unités linguistiques n'opérant jamais seules, on ne peut donc les caractériser qu'en décrivant leurs paramètres d'interaction avec d'autres unités. C'est là une difficulté majeure, qui consiste à tenter d'intégrer la relation potentielle dans l'unité, le dynamique dans le figé, le multidimensionnel dans le linéaire. Cette tâche est plus aisée lors de l'analyse des données empiriques, où les réseaux de déterminations et l'interaction des formes peuvent être mis au jour pour éclairer l'élaboration du sens construit. Le constat de l'inter-mobilisation constante des déterminations nominales et verbales par exemple, mais aussi de l'incidence des aspects lexicaux sur la détermination grammaticale (et viceversa), ou encore de la détermination verbale sur la relation de subordination (cf. Sekali 1992), sollicitent le linguiste sur la nécessité de formaliser, au niveau même des formes schématiques, la présence de ces paramètres d'interaction spécifiques.

\section{Interface entre les différents niveaux de structuration}

14 Le processus de paramétrage du sens est aussi nécessairement multidimensionnel et dynamique, puisqu'il intègre l'enchevêtrement et l'inter-mobilisation de différents niveaux (ou dimensions) de structuration, qui n'ont finalement que peu d'autonomie les 
uns par rapport aux autres : la syntaxe, la grammaire, le lexique, la prosodie, les schémas discursifs, les filtres subjectifs et situationnels etc. Les phénomènes de subordination et de coordination par exemple, ne renvoient pas aux mêmes marqueurs linguistiques selon qu'on les observe d'un point de vue syntaxique ou sémantique, si bien qu'un même marqueur comme AND/ ET peut être décrit comme marquant une coordination syntaxique mais une subordination sémantique dans des énoncés tels que 'Another step and I fire !' (cf. Sekali, ce volume). Le paramétrage du sens relationnel construit par ces unités linguistiques ne peut ainsi être envisagé que dans une analyse qui rende compte de l'interface entre ces dimensions, syntaxique et sémantique, dans l'analyse des formes.

Cet aspect multidimensionnel du sens et de son paramétrage suppose qu'on se dote d'outils d'analyse, et de modèles de représentation, qui ne hiérarchisent pas ces niveaux de structuration, et renoncent définitivement à la problématique gallinacée pour considérer les zones de contact et d'interaction entre les différentes dimensions constitutives des systèmes linguistiques. Cette démarche s'inscrit alors nécessairement dans une recherche collective.

\section{Interface entre les outils théoriques permettant d'appréhender ces différentes dimensions}

16 La démarche du paramétrage du sens est résolument inscrite dans le cadre des théories de l'énonciation. Comme cela a été précisé plus haut, les nouveaux modèles de représentations au sein de la Théorie des Opérations Prédicatives et Enonciatives, et en particulier la recherche des formes schématiques intégrant la variance dans l'identité des unités linguistiques, sont un outil fondamental dans cette optique. Paramétrer le sens suppose en effet un travail d'analyse qui porte sur des énoncés (en non des phrases), sur des corpus de textes et de discours, sur des structures linguistiques étroites et larges. Il s'agit alors de décrire et d'analyser plus que de décomposer. Le sens construit n'est pas à voir ici comme le résultat d'un calcul arithmétique de ses composantes, mais plutôt comme la résultante d'une combinaison d'opérations qui s'inter-mobilisent, et se transforment mutuellement. Les paradigmes d'interaction de paramètres sont d'abord identifiés par leur récurrence dans des corpora authentiques, puis leur mode d'interaction est caractérisé et formalisé. Dans une représentation interactionnelle des formes schématiques et des opérations, on peut considérer qu'unités lexicales et grammaticales n'ont pas lieu d'être traitées différemment, et l'esprit de la grammaire des constructions (Golberg 1995), peut également apporter des outils intéressant le paramétrage du sens à l'interface entre lexique, grammaire et syntaxe. On trouve aussi dans la grammaire instructionnelle (Victorri et Fuchs 1996 ; Col, G., Aptekman, J., Girault, S. et Victorri, B. 2010), une démarche qui vise à formaliser la variance sous forme d'un processus de convocation-évocation. Les notions de 'convocation' et d"instructions' sont donc intimement liées à la recherche des processus synergiques évoqués plus haut, et que nous cherchons à caractériser. En outre, l'organisation des représentations, et des unités discours, au sein d'entités linguistiques plus larges, influe également sur les potentialités sémantiques des unités micro-linguistiques, et devraient donc figurer comme l'un des paramètres de leur forme schématique, permettant par exemple de construire linguistiquement un 'vouloir dire' ancré dans le dire. L'inscription des micro-unités dans les macro-unités est aussi une interface à considérer dans l'élaboration des valeurs référentielles, et dans cette mesure, les théories de la pragmatique et de l'analyse du 
discours (voir en particulier Polanyi 1988; Asher et Vieu 2005) peuvent être d'un apport certain.

Pour être multidimensionnelle, la démarche du paramétrage du sens devrait alors se doter d'une métalangue et d'outils d'analyse qui puissent rendre compte des zones d'interactions et des interfaces linguistiques. On le voit, paramétrer le sens et ses potentialités est une démarche qui se veut multimodale, syncrétique, et résolument linguistique. Si la constitution d'une métalangue de l'interface est complexe, il reste qu'une démarche collective nous parait fructueuse et possible, en considérant que le point de vue sur l'objet ne recrée pas l'objet, mais que la conjonction des points de vue éclaircit l'objet, ici l'élaboration du sens, pour le rendre visible dans sa dimension de relief, à la manière d'une sorte d'hologramme sémantique.

\section{BIBLIOGRAPHIE}

ASHER, N., VIEU, L. (2005) « Subordinating and coordinating discourse relations », Lingua 115:4, pp. 591-610

COL, G., APTEKMAN, J., GIRAULT, s. et VICTORRI, B. (2010) « Compositionnalité gestaltiste et construction du sens par instructions dynamiques ", CogniTextes, Volume 5. http://cognitextes.revues.org/372 CULIOLI, A. (1990) Pour une linguistique de l'énonciation, Ophrys.

DE VOGÜÉ, S. (1992« Culioli après Benveniste : énonciation, langage, intégration », LINX, 26 : 77-108 DE VOGÜÉ, S. et PAILLARD, D. (1997) «Identité lexicale et hétérogénéité de la variation co-textuelle : le cas de suivre ». In : Co-texte et calcul du sens, C. Guimier, éd., Caen, Presses Universitaires de Caen.

FRANCKEL, J-J., PAILLARD, D., et SAUNIER, E. (1997) « Modes de régulation de la variation sémantique d'une unité lexicale : le cas du verbe passer ». In : La locution : entre lexique, syntaxe et pragmatique, coll. St-Cloud, publication de l'INALF, Paris : Klincksieck, pp. 49-68.

FRANCKEL, J-J et PAILLARD, D. (1998) « Aspects de la théorie d'Antoine Culioli », Langages, 32-129 : $52-63$.

GOLDBERG, A. (1995) Constructions - a Construction Grammar Approach to Argument Structure, Chicago: University of Chicago Press.

POLANYI, L. (1988) « A formal model of the structure of discourse » Journal of Pragmatics 12: 601-638.

SEKALI, M. (1992) «Subordination temporelle et subordination subjective : l'exemple de since », Travaux du CERLICO, Subordination/Subordinations, 5 :105-126.

VICTORRI, B. et FUCHS, C. (1996) La Polysémie, Hermès. 
AUTEUR

MARTINE SEKALI

Université Paris Ouest Nanterre 